\title{
Combined Serological Detection of Circulating Angiostrongylus vasorum Antigen and Parasite-specific Antibodies in Dogs from Hungary
}

Manuela Schnyder ${ }^{\circledR} \bowtie$, Roland Schaper², Zoltán Lukács ${ }^{3}$, Sándor Hornok ${ }^{4}$, Róbert Farkas ${ }^{4}$

1 Institute of Parasitology, Vetsuisse Faculty, University of Zurich, 8057 Zurich, Switzerland

2 Bayer Animal Health GmbH, 51368 Leverkusen, Germany

3 Bayer Hungaria Kft., 1123 Budapest, Hungary

4 Department of Parasitology and Zoology, Faculty of Veterinary Science Budapest, 1078 Budapest, Hungary

Corresponding author:

\section{Manuela Schnyder}

E-mail: manuela.schnyder@uzh.ch

\section{Abstract}

The occurrence of the nematode Angiostrongylus vasorum, also known as the French heartworm, is increasingly being reported from various European countries. The adults of this parasite species live in the pulmonary arteries and right cardiac ventricle of wild canids and domestic dogs. Larval stages and eggs in the lungs induce inflammatory verminous pneumonia, causing severe respiratory disease in dogs. Furthermore, haematological and neurological signs and even death may occur. In Hungary, A. vasorum has been identified in red foxes, golden jackals and in two dogs and some slugs. In this first large-scale survey, 1247 sera from pet dogs were collected and tested by an ELISA for the detection of circulating antigen of A. vasorum and by a separate
ELISA to detect specific antibodies against the parasite. A total of $1.36 \%(\mathrm{n}=17,95 \%$ confidence intervals, CI: $0.80-2.17 \%$ ) of the animals were positive in both ELISAs, while $1.76 \%$ ( $n=22$, CI: $1.11-2.66 \%$ ) of the tested dogs were antigen-positive only and $2.73 \%$ ( $\mathrm{n}=34$, CI: $1.90-3.79 \%$ ) were positive for specific antibodies only. Regions with antigen- and antibodypositive animals overlapped and were distributed over nearly the whole sampled areas of the country. A considerable number of cases was observed in Budapest and also in the southern part of the country bordering Croatia, while in the most eastern part bordering Ukraine no positive samples were detected. These results confirm the endemic occurrence of $A$. vasorum in dogs originating from different parts of Hungary and the significant advantages of $A$. vasorum serology in epidemiological studies. 


\section{Introduction}

Canine angiostrongylosis is a potentially fatal disease caused by the metastrongylid nematode Angiostrongylus vasorum, which is increasingly being reported throughout Europe. The adult parasite lives in the pulmonary arteries and right cardiac ventricle of dogs, foxes and other wild carnivores, which are infected through the ingestion of obligatory intermediate hosts (snails or slugs) (Guilhon and Bressou 1960; Guilhon and Cens 1973) or paratenic hosts (Bolt et al. 1993) containing the infectious third stage larvae. Clinical signs in dogs most frequently include respiratory signs such as coughing and dyspnoea, but a broad range of further signs indicating coagulopathies or neurological dysfunctions (Chapman et al. 2004; Staebler et al. 2005; Wessmann et al. 2006; Koch and Willesen 2009), may be the signs most obvious to clinicians and animal owners. This variability and the fact that the disease is often in an advanced stage when noticed by the animal owners make the diagnosis of A. vasorum infections challenging but important: the sooner an appropriate anthelmintic treatment is initiated, the better for the clinical recovery of the dogs and limitation of damage, especially to lung tissues (Dennler et al. 2011; Schnyder et al. 2009).

A fundamental role in the early diagnosis of infections is played by awareness of the local occurrence of the parasite and, correspondingly, disease awareness among vets and animal owners. The endemic occurrence of $A$. vasorum is obviously linked to the presence of final hosts, among which red foxes represent an important wild reservoir, and the intermediate hosts, i.e. a broad range of snail and slug species (Eckert and Lämmler 1972; Ferdushy et al. 2009; Patel et al. 2014). Interestingly, although both final and intermediate hosts are widely present, the distribution of foxes and dogs affected by A. vasorum is scattered (Morgan 2014), and the reasons for this patchy distribution are not yet fully understood. Accordingly, it is particularly important to have good knowledge of the occurrence of
A. vasorum in a specific catchment area of a veterinary practice. Southern France (Guilhon and Cens 1969; Bourdeau 1993), Ireland (Dodd 1973), south-east England and Wales (Jacobs and Prole 1975; Simpson and Neal 1982) and Denmark (Bolt et al. 1992) have traditionally been endemic foci of A.vasorum; in the past two decades there has additionally been a sharp increase in reports from other countries in central Europe (Staebler et al. 2005; Barutzki and Schaper 2009), the Iberian peninsula (Segovia et al. 2004; Manas et al. 2005; Alho et al. 2014;) and also from Italy (Della Santa et al. 2002; Guardone et al. 2013), Greece (Papazahariadou et al. 2007) and Croatia (Rajkovic-Janje et al. 2002). Very recent case reports from eastern European countries describe the presence of the parasite in dogs from Poland (Demiaszkiewicz et al. 2014) and Slovakia (Miterpakova et al. 2014). In Hungary, which has common borders with Slovakia and Croatia, A.vasorum was first identified in 5 out of 100 red foxes shot in the western and southern counties of the country (Sréter et al. 2003), in two golden jackals (Canis aureus) (Takács et al. 2013) and also in two dogs and some slugs from close to the Croatian border diagnosed as being positive (Majoros et al. 2010).

The long-standing method for confirmation of clinical suspicion of dogs infected with $A$. vasorum, once the dogs are patent at approximately 40-57 days post infection (Guilhon and Cens 1973; Schnyder et al. 2010), is the detection of first stage larvae in faeces performed with the Baermann-Wetzel technique (Deplazes et al. 2013). However, by the time dogs start to be Baermann-positive, damage to the lung parenchyma is already present (Dennler et al. 2011; Guilhon and Cens 1969; Neff 1971). In addition, copromicroscopic methods have limitations concerning sensitivity and specificity due to variable larval excretion and the presence of other lungworm larvae (e.g. of Crenosoma vulpis) that may be excreted. Among the more recently applied techniques such as PCR (Jefferies et al. 2009; AlSabi et al. 2010) and serological methods (Cury et al. 1996; Verzberger-Epshtein et al. 2008) for 
Table 1: Serological results of 1247 dog samples from Hungary tested for the presence of circulating antigens of $A$. vasorum and of specific antibodies against $A$. vasorum.

\begin{tabular}{|c|c|c|c|}
\hline & Positive samples $(\mathbf{n})$ & $\%$ & $95 \%$ confidence intervals \\
\hline Antibody-positive & 51 & 4.09 & $3.06-5.34$ \\
\hline Antibody-positive only & 34 & 2.73 & $1.90-3.79$ \\
\hline Antigen-positive & 39 & 3.13 & $2.23-4.25$ \\
\hline Antigen-positive only & 22 & 1.76 & $1.11-2.66$ \\
\hline Antibody- and antigen-positive & 17 & 1.36 & $0.80-2.17$ \\
\hline
\end{tabular}

the detection of infected animals, serological methods have been shown to be highly suitable for both individual and population studies (Guardone et al. 2013; Schnyder et al. 2013a). Notably, mass screening with ELISAs is a valid and affordable method for investigation of large dog populations.

The aim of this study was to perform a seroepidemiological survey for the detection of circulating antigens of $A$. vasorum and of specific antibodies in sera of dogs originating from Hungary in order to increase knowledge of the presence of A. vasorum in dogs from this country.

\section{Materials and methods}

Sera of 1247 dogs from Hungary were collected from dogs attending veterinary clinics for different reasons. Subsamples were sent to the Institute of Parasitology, Vetsuisse Faculty, University of Zurich, Switzerland and were analysed for the presence of circulating $A$. vasorum antigens using monoclonal and polyclonal antibodies in a sandwich ELISA with a sensitivity of $95.7 \%$ and a specificity of $94.0 \%$, as previously described (Schnyder et al. 2011). Additionally, a sandwich ELISA (sensitivity $81.0 \%$, specificity $98.8 \%$ ) using A. vasorum adult somatic antigen purified by monoclonal antibodies (mAb Av 5/5) was used for specific antibody detection (Schucan et al. 2012). Test thresholds were regionally determined with 300 randomly selected samples based on the mean value of optical density $\left(\mathrm{A}_{405 \mathrm{~nm}}\right)$ plus 3 (antigen detection) or 4 (antibody detection) standard deviations. All test runs included a background control, a conjugate control, three positive control sera from three experimentally infected dogs and two negative control sera from uninfected dogs.

The collected data were analysed by a geographical information system (GIS) using the program RegioGraph 10 (GfK GeoMarketing, Bruchsal, Germany) to visualize the regional distribution of collected and analysed serum samples and A. vasorum antigen- and/or antibody-positive samples. The locations of positive samples were displayed on maps with administrative and postcode boundaries based on the three-digit postcodes of Hungary as points of reference.

Excel 2007 for Windows (Microsoft Corporation, Redmond, USA) was used to calculate the prevalence values and the $95 \%$ confidence interval (CI) of prevalence values.

\section{Results}

The seropositivity of all tested samples is summarised in Table 1. A total of $1.36 \%(n=17$, confidence intervals, CI: $0.80-2.17 \%$ ) of the animals were positive in both ELISAs, while $1.76 \%$ of the tested dogs were only antigen-positive and $2.73 \%$ were positive for specific antibodies only. The locations of positive sera are shown in Fig. 1a-c. Regions with antigen- and antibody-positive animals overlapped and were distributed over nearly the whole sampled areas of the country. The area of Budapest is 


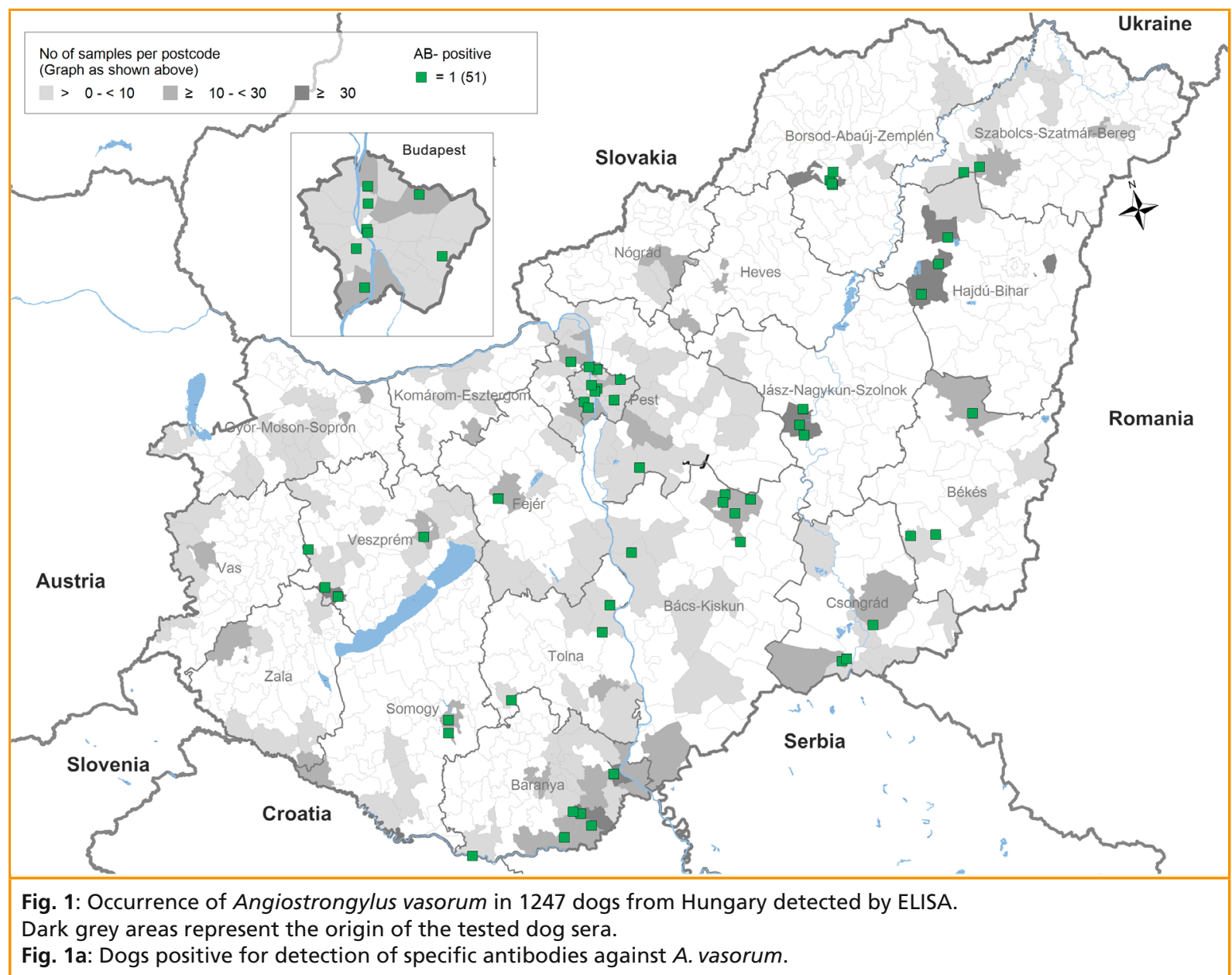

enlarged, showing a considerable number of cases. An accumulation of cases can also be observed in the southern part of the country bordering Croatia, while in the most eastern part bordering Ukraine no positive samples were detected.

\section{Discussion}

With $1.36 \%$ of the examined dogs being positive in both ELISAs, the prevalence in Hungary is significantly higher than that found for Germany (Schnyder et al. 2013a), Italy (Guardone et al. 2013) or Poland (Schnyder et al. 2013b), which were all between 0.3 and $0.5 \%$ and all obtained using the same validated procedures. More than $4 \%$ of the dogs were positive for specific antibodies against A.vasorum, indicating parasite exposure: these dogs may have been sampled during the first five weeks after an A. vasorum infection, when antigen detection is still negative, or the dogs were parasite-free but still antibody-positive after anthelmintic treatment or natural clearance of the infection (Schnyder et al. 2013a). The procedures used, i.e. the combination of both ELISAs, again confirmed their utility for mass screening of dog populations. In contrast to Poland, where testing of more than 3000 sera revealed for the first time the presence of A.vasorum in the country (Schnyder et al. 2013b) and positive foxes were identified subsequently (Demiaszkiewicz et al. 2014), the occurrence of A. vasorum in Hungary had already been reported. The parasite had first been described in foxes: 5 of 100 red foxes (Vulpes 


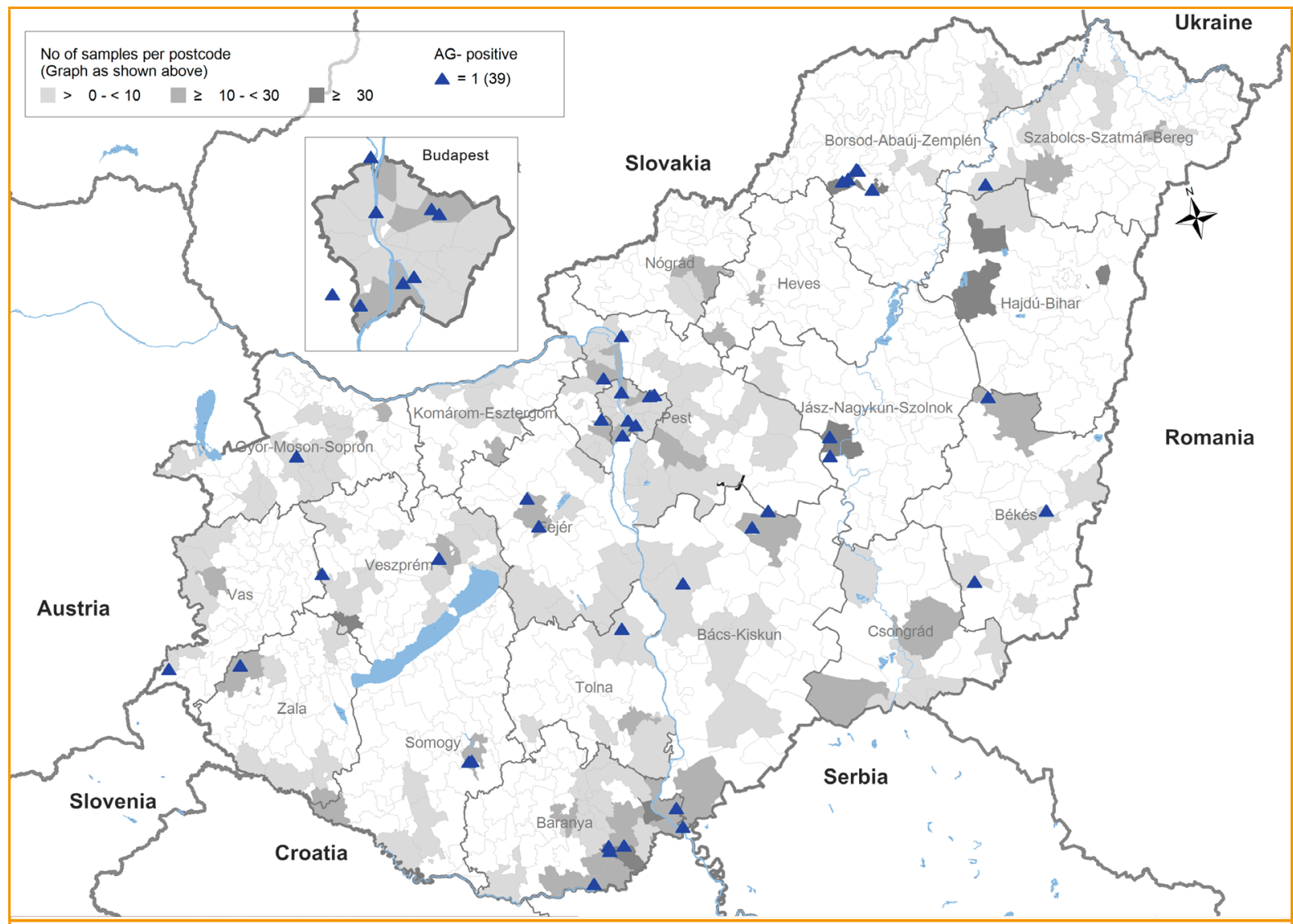

Fig. 1b: Dogs positive for detection of circulating antigen of $A$. vasorum.

vulpes) from 16 Hungarian counties dissected in 2002 were positive at necropsy, with four foxes having low worm burdens (1-7 adult specimens) and a single fox having a moderate worm burden (8-14 specimens). These foxes originated from the southern and western parts of the country (Sréter et al. 2003), while none of the surrounding countries had reported cases of $A$. vasorum at that stage. Two asymptomatic dogs from south-western Hungary were diagnosed positive for A.vasorum based on positive faecal samples (Majoros et al. 2010). These cases were followed more recently by further reports of $A$. vasorum in wild carnivores, for instance in 2 out of 10 golden jackals (Canis aureus) (Takács et al. 2013). In a current analysis of the environmental factors having an impact on the distribution of lung worms in foxes based on the dissection of 937 animals, an average prevalence of $17.9 \%$ was detected, with high variations from $0-59.4 \%$ between counties (Tolnai et al. 2015). Interestingly, $A$. vasorum was nearly absent in the eastern and central-southern part of the country, while C.vulpis was also detected in the most eastern part. This latter lung worm species, which is located in the bronchi and bronchioles of dogs and foxes, is also a cause of respiratory diseases and uses snails and slugs as intermediate hosts (Stockdale and Hulland 1970; Unterer et al. 2002). Its prevalence in foxes in 2013/14 (24.6\%) (Tolnai et al. 2015) was identical to that detected in $2002(24 \%)$ (Sréter et al. 2003). Therefore, if the presence of C. vulpis is long-standing and stable in Hungary, data from infected foxes and dogs show that A. vasorum is a more recent phenomenon. The occurrence of canine angiostrongylosis is actually less frequent, and in foxes its dissemination is not 


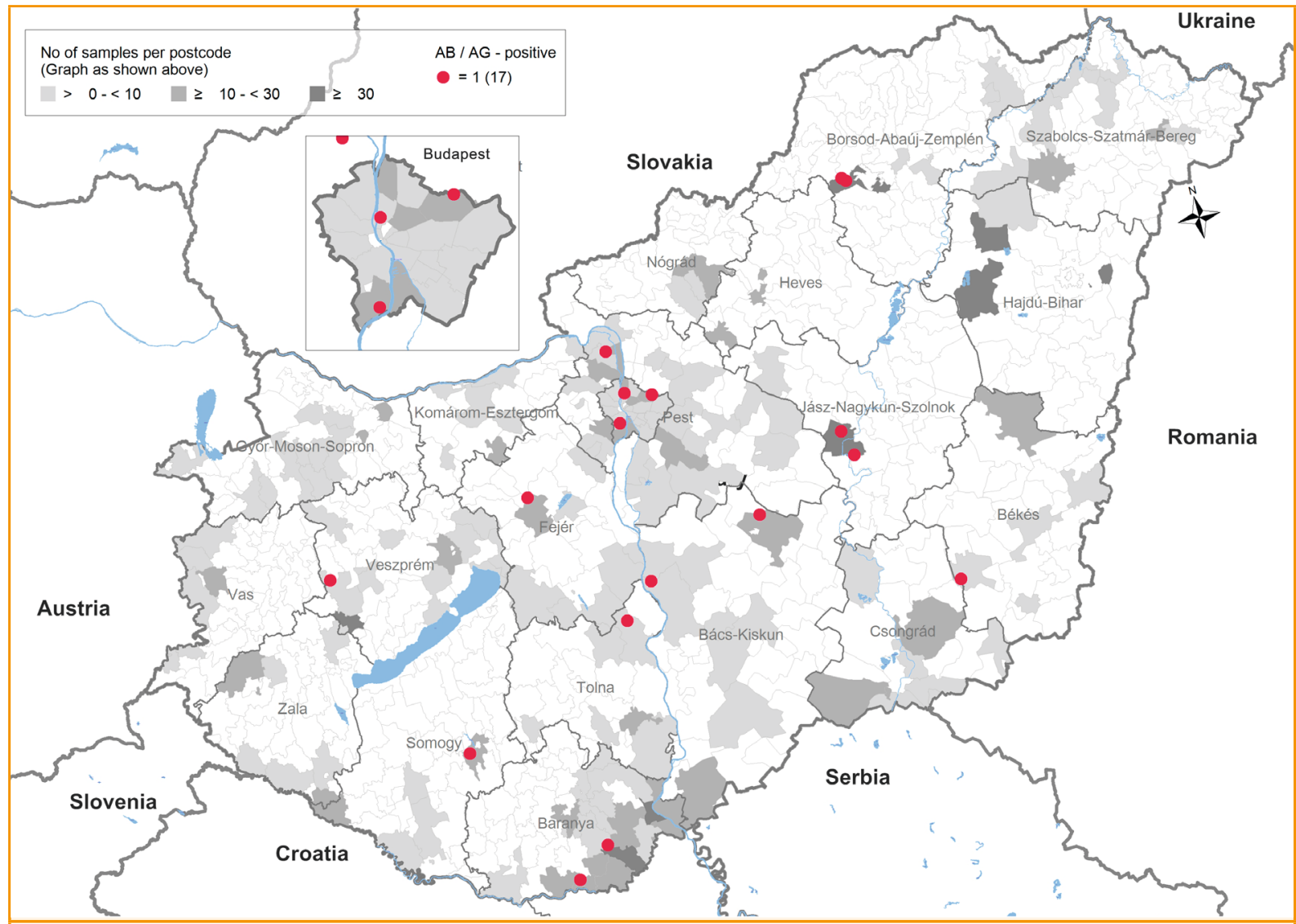

Fig. 1c: Combined testing for detection of $A$. vasorum antigen and specific antibodies.

as broad as for C.vulpis, although they may share the same intermediate hosts and mollusc species are broadly distributed in Hungary (Sólymos and Fehér 2005).

During the last few decades, reports of animals affected by A. vasorum animals have become more frequent and various reasons for the apparent spread of lung worms have been discussed. Regional climate changes in vector epidemiology and movements in animal populations were taken into account (Morgan et al. 2009; Tolnai et al. 2015). Dog data collected in this study support the gradual advancement of $A$. vasorum in eastern Europe: in Hungary the parasite has been detected in parts of the country bordering Croatia in the south and Slovakia in the north, where the parasite has been already reported (Miterpakova et al. 2014; Rajkovic-Janje et al. 2002), while little is known from the other neighbouring countries (Austria, Romania, Slovenia, Serbia and Ukraine).

The evidence of a higher prevalence of all important lungworms (A.vasorum, C.vulpis and Eucoleus aerophilus) in foxes (Sréter et al. 2003; Tolnai et al. 2015) compared to dogs (Koch and Willesen 2009) indicates that wildlife, in the absence of obvious geographical barriers, plays an important role in expansion and establishment of these parasites. Hungary, like many other countries, has implemented successful rabies control programmes (Solymosi et al. 2002), which implies a massive increase in the fox population. Therefore, it is of general acceptance that foxes contribute essentially to the local distribution and establishment of $A$. vasorum, while infected dogs that travel long distances may contribute to a broader spread beyond connected endemic areas. 
The major clinical impact of $A$. vasorum infections in dogs means that it is of essential importance to increase awareness of the occurrence of A. vasorum among clinicians and dog owners, particularly in previously unreported areas bordering endemic regions, with the aim of preventing fatal cases of canine angiostrongylosis. Newly developed, rapid and easy-to-use commercial devices for use in veterinary practices (Schnyder et al. 2014) and adequate metaphylactic and prophylactic anthelmintic treatments (Conboy 2001; Willesen et al. 2007; Schnyder et al. 2009) allow early diagnosis and consequently a good prognosis for dogs affected by A. vasorum.

\section{References}

Al-Sabi MN, Deplazes P, Webster P, Willesen JL, Davidson RK, Kapel CM (2010) PCR detection of Angiostrongylus vasorum in faecal samples of dogs and foxes. Parasitol Res $107: 135-140$

Alho AM, Schnyder M, Meireles J, Belo S, Deplazes P, Madeira de Carvalho L (2014) Preliminary results on the seroprevalence of Angiostrongylus vasorum and co-infection with Dirofilaria immitis in shelter dogs from Portugal. Paras \& Vectors 7 Suppl 1:O26

Barutzki D, Schaper R, (2009) Natural infections of Angiostrongylus vasorum and Crenosoma vulpis in dogs in Germany (2007-2009). Parasitol Res 105 Suppl 1: S39-48

Bolt G, Monrad J, Henriksen P, Dietz HH, Koch J, Bindseil E, Jensen AL (1992) The fox (Vulpes vulpes) as a reservoir for canine angiostrongylosis in Denmark. Field survey and experimental infections. Acta Vet Scand 33: 357-362

Bolt G, Monrad J, Henriksen P, Dietz HH, Koch J, Bindseil E, Jensen AL (1993) The common frog (Rana temporaria) as a potential paratenic and intermediate host for Angiostrongylus vasorum. Parasitol Res 79, 428-430

Bourdeau P (1993) L’angiostrongylose canine. Receuil Méd Vét 169: 401-407

Chapman PS, Boag AK, Guitian J, Boswood A (2004) Angiostrongylus vasorum infection in 23 dogs (1999-2002). J Small Anim Practice 45: 435-440

\section{Ethicall standards}

All institutional and national guidelines for the care and use of laboratory animals were followed. Funding: The study was partially funded by Bayer Animal Health.

\section{Conflict of interest}

M. Schnyder, S. Hornok and R. Farkas declare no conflict of interest, R. Schaper and Z. Lukács are employed by Bayer Animal Health.

\section{Acknowledgements}

We are grateful to Kathrina Stebler and Katja Huggel from the Institute of Parasitology in Zurich and Mónika Gyurkovszky from the Department of Parasitology and Zoology at the Faculty of Veterinary Science in Budapest for their extremely valuable support.

Conboy G (2004) Natural infections of Crenosoma vulpis and Angiostrongylus vasorum in dogs in Atlantic Canada and their treatment with milbemycin oxime. Vet Rec 155, 16-18

Cury MC, Lima WS, Vitor RWA (1996) Enzyme-Linked Immunosorbent Assay (ELISA) for the diagnosis of Angiostrongylus vasorum (Baillet, 1866) infection in dogs. Revue Méd Vét 147: 525-530

Della Santa D, Citi D, Marchetti V, Nardoni S (2002) Infestione da Angiostrongylus vasorum nel cane: review della letteratura e presentazione di un caso clinico. Veterinaria 16: $9-14$

Demiaszkiewicz AW, Pyziel AM, Kuligowska I, Lachowicz J (2014) The first report of Angiostrongylus vasorum (Nematoda; Metastrongyloidea) in Poland, in red foxes (Vulpes vulpes). Acta Parasitol 59: 758-762

Dennler M, Makara M, Kranjc A, Schnyder M, Ossent P, Deplazes P, Ohlerth S, Glaus TM (2011) Thoracic computed tomography findings in dogs experimentally infected with Angiostrongylus vasorum. Vet Radiol Ultrasound 52: $289-294$

Deplazes P, Eckert J, Samson-Himmelstjerna, Gv, Zahner H (2013) Lehrbuch der Parasitologie für die Tiermedizin (Stuttgart, Enke Verlag).

Dodd K (1973) Angiostrongylus vasorum (Baillet, 1866) infestation in a greyhound kennels. Vet Rec 92: 195-197 
Eckert J, Lämmler G (1972) Angiostrongylose bei Mensch und Tier. Parasitol Res 39: 303-322

Ferdushy T, Kapel CM, Webster P, Al-Sabi MN, Gronvold J (2009) The occurrence of Angiostrongylus vasorum in terrestrial slugs from forests and parks in the Copenhagen area, Denmark. J Helminthol 83: 379-383

Guardone L, Schnyder M, Macchioni F, Deplazes P, Magi M (2013) Serological detection of circulating Angiostrongylus vasorum antigen and specific antibodies in dogs from central and northern Italy. Vet Parasitol 192: 192-198.

Guilhon J, Bressou C (1960) Rôle des Limacidés dans le cycle évolutif d'Angiostrongylus vasorum (Baillet, 1866). C R Acad Sc 251: 2252-2253

Guilhon J, Cens B (1969) [Migrations and evolution of Angiostrongylus vasorum (Baillet, 1866) in dogs (in French)]. Comptes Rendus Hebdomadaires des Seances de l'Academie des Sciences Naturelles 269: 2377-2380.

Guilhon J, Cens B (1973) Angiostrongylus vasorum (Baillet, 1866): Étude biologique et morphologique. Ann Parasitol Hum Comp 48: 567-596

Jacobs DE, Prole JH (1975) Angiostrongylus vasorum and other nematodes in British greyhounds. Vet Rec 96: 180

Jefferies R, Morgan ER, Shaw SE (2009) A SYBR green realtime PCR assay for the detection of the nematode Angiostrongylus vasorum in definitive and intermediate hosts. Vet Parasitol 166: 112-118

Koch J, Willesen JL (2009) Canine pulmonary angiostrongylosis: An update. Vet J 179: 348-359

Majoros G, Fukar O, Farkas R, (2010) Autochtonous infection of dogs and slugs with Angiostrongylus vasorum in Hungary. Vet Parasitol 174: 351-354.

Manas S, Ferrer D, Castella J, Maria Lopez-Martin J (2005) Cardiopulmonary helminth parasites of red foxes (Vulpes vulpes) in Catalonia, northeastern Spain. Vet J 169: 118-120

Miterpakova M, Hurnikova Z, Zalewski AP (2014) The first clinically manifested case of angiostrongylosis in a dog in Slovakia. Acta Parasitol 59: 661-665

Morgan ER (2014) Canine pulmonary angiostrongylosis: can a worm change its spots? Vet Rec 175: 116-117

Morgan ER, Jefferies R, Krajewski M, Ward P, Shaw SE (2009) Canine pulmonary angiostrongylosis: the influence of climate on parasite distribution. Parasitol Int 58: 406-410
Neff H, (1971) Experimentelle Infektionen von Hunden mit Angiostrongylus vasorum (Nematoda). Dissertation, Universität Zürich

Papazahariadou A, Founta A, Papadopoulos E, Chliounakis S, Antoniadou-Sotiriadou K, Theodorides Y (2007) Gastrointestinal parasites of shepherd and hunting dogs in the Serres Prefecture, Northern Greece. Vet Parasitol 148: $170-173$.

Patel Z, Gill AC, Fox MT, Hermosilla C, Backeljau T, Breugelmans K, Keevash E, McEwan C, Aghazadeh M, ElsonRiggins JG (2014) Molecular identification of novel intermediate host species of Angiostrongylus vasorum in Greater London. Parasitol Res 113: 4363-4369

Rajkovic-Janje R, Marinculic A, Bosnic S, Benic M, Vinkovic B, Mihaljevic Z (2002) Prevalence and seasonal distribution of helminth parasites in red foxes (Vulpes vulpes) from the Zagreb County (Croatia). Z Jagdwiss 48: 151-160

Schnyder M, Fahrion A, Ossent P, Kohler L, Webster P, Heine J, Deplazes P (2009) Larvicidal effect of imidacloprid/ moxidectin spot-on solution in dogs experimentally inoculated with Angiostrongylus vasorum. Vet Parasitol 166: $326-332$

Schnyder M, Fahrion A, Riond B, Ossent P, Webster P, Kranjc A, Glaus T, Deplazes P (2010) Clinical, laboratory and pathological findings in dogs experimentally infected with Angiostrongylus vasorum. Parasitol Res 107: 1471-1480

Schnyder M, Tanner I, Webster P, Barutzki D, Deplazes P (2011) An ELISA for sensitive and specific detection of circulating antigen of Angiostrongylus vasorum in serum samples of naturally and experimentally infected dogs. Vet Parasitol 179: $152-158$.

Schnyder M, Schaper R, Bilbrough G, Morgan ER, Deplazes P, (2013a) Seroepidemiological survey for canine angiostrongylosis in dogs from Germany and the UK using combined detection of Angiostrongylus vasorum antigen and specific antibodies. Parasitology 140: 1442-1450

Schnyder M, Schaper R, Pantchev N, Kowalska D, Szwedko A, Deplazes P, (2013b) Serological detection of circulating Angiostrongylus vasorum antigen- and parasite-specific antibodies in dogs from Poland. Parasitol Res 112 Suppl 1: 109-117

Schnyder M, Stebler K, Naucke TJ, Lorentz S, Deplazes P (2014) Evaluation of a rapid device for serological in-clinic diagnosis of canine angiostrongylosis. Paras \& Vectors 7: 72

Schucan A, Schnyder M, Tanner I, Barutzki D, Traversa D, Deplazes P (2012) Detection of specific antibodies in dogs infected with Angiostrongylus vasorum. Vet Parasitol 185: $216-224$. 
Segovia JM, Torres J, Miquel J (2004) Helminth parasites of the red fox (Vulpes vulpes L., 1758) in the Iberian Peninsula: an ecological study. Acta Parasitologica 49: 67-79

Simpson VR, Neal C (1982) Angiostrongylus vasorum infection in dogs and slugs. Vet Rec 111: 303-304

Sólymos P, Fehér Z (2005) Conservation prioritization based on the distribution of land snails in Hungary. Conserv Biol 19: $1084-1094$

Solymosi N, Reiczigel J, Edelyi K, Banhidy J, Foldi Z, Bodis K (2002) Spatial analysis of rabies cases in foxes in Hungary between 1990 and 2001: a preliminary report. Stud Health Technol Inform 90: 770-773

Sréter T, Széll Z, Marucci G, Pozio E, Varga I (2003) Extraintestinal nematode infections of red foxes (Vulpes vulpes) in Hungary. Vet Parasitol 115: 329-334

Staebler S, Ochs H, Steffen F, Naegeli F, Borel N, SieberRuckstuhl N, Deplazes P (2005) Autochthonous infections with Angiostrongylus vasorum in dogs in Switzerland and Germany (in German). Schweiz Arch Tierheilkde 147:121-127

Stockdale PH, Hulland TJ, (1970) The pathogenesis route of migration, and development of Crenosoma vulpis in the dog. Pathol Vet 7: 28-42

Takács A, Szabo L, Juhász L, Takács AA, Lanszki J, Takács PT, Heltai M (2013) Data on the parasitological status of golden jackal (Canis aureus l., 1758) in Hungary. Acta Vet Hung: 1-9.
Tolnai Z, Széll Z, Sréter T (2015) Environmental determinants of the spatial distribution of Angiostrongylus vasorum, Crenosoma vulpis and Eucoleus aerophilus in Hungary. Vet Parasitol 207: 355-358

Unterer S, Deplazes P, Arnold P, Flückiger M, Reusch CE, Glaus TM (2002) Spontaneous Crenosoma vulpis infection in 10 dogs: laboratory, radiographic and endoscopic findings. Schweiz Arch Tierheilkd 144: 174-179

Verzberger-Epshtein I, Markham RJ, Sheppard JA, Stryhn H, Whitney H, Conboy GA (2008) Serologic detection of Angiostrongylus vasorum infection in dogs. Vet Parasitol 151: $53-60$

Wessmann A, Lu D, Lamb CR, Smyth B, Mantis P, Chandler K, Boag A, Cherubini GB, Cappello R (2006) Brain and spinal cord haemorrhages associated with Angiostrongylus vasorum infection in four dogs. Vet Rec 158: 858-863

Willesen JL, Kristensen AT, Jensen AL, Heine J, Koch J (2007) Efficacy and safety of imidacloprid/moxidectin spot-on solution and fenbendazole in the treatment of dogs naturally infected with Angiostrongylus vasorum (Baillet, 1866). Vet Parasitol 147:258-264. 
